\title{
Computed tomography angiography to identify the cause of a transient ischemic attack versus irradiating patients without cause (or even a TIA)
}

\author{
Jeffrey J. Perry, MD, MSc ${ }^{* \dagger}$; Mukul Sharma, MD, MSc ${ }^{\S}$
}

The article by Bibok and colleagues addresses the issue of balancing the need to urgently image the vessels of high-risk transient ischemic attack (TIA) patients versus the identification of low-risk patients for whom this imaging can be withheld (or delayed). ${ }^{1}$ Imaging blood vessels helps determine TIA/stroke etiology, which may lead to specific therapy. It also assists with predicting the prognosis for recurrent cerebrovascular events. ${ }^{2}$ For TIA patients, angiography of the neck arteries identifies those with critical carotid artery stenosis (i.e., $\geq 50 \%$ ). Early carotid artery revascularization (i.e., carotid endarterectomy or stent) is critical for symptomatic critical carotid stenosis (i.e., $\geq 50 \%$ ). ${ }^{3}$ When intervention for symptomatic stenosis is performed within 2 weeks, the number needed to treat (NNT) to prevent death or subsequent stroke is 6 . The NNT is even smaller for $70 \%-99 \%$ stenosis (NNT of 3.3). Yet, the NNT increases to 125 if the intervention is more than 12 weeks post-TIA and the benefit is no longer statistically significant. While baseline patient status and surgical risks must be taken into consideration, the benefits of carotid revascularization to prevent subsequent stroke are time sensitive. In addition, imaging of the entire vascular tree may identify rarer conditions that benefit from specific treatment (e.g., aortic or another artery dissection, cerebral angiitis).

While there is a clear need for early arterial imaging for the above-mentioned reasons, there is likewise a problem with imaging patients without TIA. Symptoms attributable to another cause account for up to $40 \%-60 \%$ of patients diagnosed with a TIA or minor stroke in emergency departments (EDs). As such, many patients do not need computed tomography angiography (CTA) as they do not have cerebral ischemia. Hence, a tool to discriminate stroke mimics and TIA/stroke is necessary. Given the radiation exposure, contrast risks, and contribution to ED crowding, modalities such as rapid outpatient carotid Duplex Doppler ultrasound may be a better option to test moderate to low risk patients. However, welltrained sonographers who routinely perform this examination are needed to obtain reliable results, and the wait time for this test may be prohibitive in some centres.

In this paper, Bibok and colleagues suggest ordering a CTA according to an automated tool based on a regression model. ${ }^{1}$ Although this risk assessment tool appears to have the potential to improve the decision-making around a TIA (they identified 22 of 23 patients undergoing a carotid artery revascularization), it remains uncertain how effective this tool will be in clinical practice. This study was conducted retrospectively, at one site (incorporating three EDs), and only included patients deemed as a high enough risk to be referred to the stroke prevention clinic. As such, the true performance of this rule cannot be fully assessed for all patients diagnosed with a TIA in the ED. Nevertheless, their objective to improve the use of CTA is important. At present, it is likely that patients deemed to be at high risk (i.e., calculated to likely have a TIA as determined by their tool that assesses for no bilateral signs, unilateral weakness, or speech disturbance with risk factors, amongst other variables) should have a CTA. Therefore, those deemed to be at low risk must still be considered carefully.

From the Departments of *Emergency Medicine; †School of Epidemiology, Public Health and Preventive Medicine, University of Ottawa; $¥$ Clinical Epidemiology Program, Ottawa Hospital Research Institute, Ottawa, ON; and the §Population Health Research Institute and Division of Neurology, McMaster University, Hamilton, ON.

Correspondence to: Dr. Jeffrey J. Perry, Clinical Epidemiology Unit, F647, The Ottawa Hospital, 1053 Carling Avenue, Ottawa, ON K1Y 4E9; Email: jperry@ohri.ca

(C) Canadian Association of Emergency Physicians

CJEM 2019;21(3):313-314

DOI 10.1017/cem.2019.27 
The 2018 Heart and Stroke Foundation of Canada guidelines categorize as "very high risk," patients presenting within 48 hours of a suspected TIA with one of the following clinical pictures: 1) transient, fluctuating, or persistent unilateral weakness; 2) transient, fluctuating, or persistent language/speech disturbance; or 3) fluctuating or persistent symptoms without weakness or language/speech disturbance (e.g., hemibody sensory symptoms, monocular vision loss, hemifield vision loss, binocular diplopia, dysarthria, dysphagia, or ataxia). ${ }^{4}$ For these patients, it is suggested that they be seen immediately in an ED with the capability of brain imaging. They recommend brain imaging (computed tomography or magnetic resonance imaging [MRI]) and vascular imaging (CTA or magnetic resonance angiography from aortic arch to vertex) within 24 hours. In this paper, the authors assess many of the clinical features included in these guidelines; hence, it is not clear how their tool compares with this guideline. The discriminative ability of this tool should also be compared with tools specifically designed to identify those at high risk of early recurrence, such as the Canadian TIA Score or the $\mathrm{ABCD}^{2}$ Score. ${ }^{5,6}$

Patients considered to be at low risk of a TIA with one of the common stroke mimics (e.g., migraine and peripheral vestibulopathies) should not have angiography in the ED. An improved ability to recognize these mimics should be a priority. In this regard, the following clinical principles are pertinent. Patients with transient neurological deficits lasting for $<1$ hour and followed by a headache, or associated with a headache, with a history of migraine (with or without aura) are likely experiencing a migraine. Patients with recurrent symptoms with movement of short lasting isolated vertigo (i.e., $<2$ minutes) are likely to have benign positional vertigo. Those with longer lasting or continuous vertigo need an assessment for possible posterior circulation cerebral ischemia versus vestibular neuritis. For this, it is important to 1 ) assess for other deficits (e.g., gait, coordination, cranial nerves) and 2) assess HINTS exam. These two assessments will determine their risk. It is also important to realize that a CTA is not usually beneficial to identify posterior circulation ischemia. MRI imaging may assist in this endeavour, but it is less available than a CTA. Further, management will be antiplatelets in these patients while investigating for a cardioembolic source. These patients will not have a carotid artery (i.e., anterior circulation) cause and therefore not need urgent revascularization.

In summary, Bibok and colleagues identify an important issue facing Canadian EDs. Imaging of patients with a presumptive diagnosis of TIA is critical for those with large vessel etiologies. Conversely, with approximately half of all patients diagnosed with TIA having a stroke mimic, some discretion is warranted. Their proposed tool may improve the approach, but prospective validation in multiple centres is required, followed by evidence that routine use is associated with better process and patient outcomes before widespread implementation. Until then, consideration should be given to highrisk patients, given it did identify more critical carotid stenosis patients than the usual practice.

Keywords: Computed tomography angiography (CTA), stroke, transient ischemic attack (TIA)

Competing interests: None declared.

\section{REFERENCES}

1. Bibok MB, Votova K, Balshaw RF, et al. Retrospective evaluation of a clinical decision support tool for effective computed tomography angiography utilization in urgent brain imaging of suspected TIA/minor stroke in the emergency department. C7EM 2019;21(3):343-51.

2. Kamal K, Hill MD, Blacquiere DP, et al. Rapid assessment and treatment of transient ischemic attacks and minor stroke in Canadian emergency departments: time for a paradigm shift. Stroke 2015;46:2987-90.

3. Rothwell PM, Eliasziw M, Gutnikov SA, et al. Endarterectomy for symptomatic carotid stenosis in relation to clinical subgroups and timing of surgery. Lancet 2004;363:915-24.

4. Boulanger JM, Lindsay MP, Gubitz G, et al. Canadian stroke best practice recommendations for acute stroke management: prehospital, emergency department, and acute inpatient stroke care, 6th edition, update 2018. Int 7 Stroke 2018;13:949-84.

5. Perry JJ, Sharma M, Sivilotti ML, et al. A prospective cohort study of patients with transient ischemic attack to identify high-risk clinical characteristics. Stroke 2014;45:92-100.

6. Johnston SC, Rothwell PM, Nguyen-Huynh MN, et al. Validation and refinement of scores to predict very early stroke risk after transient ischaemic attack. Lancet 2007;369:283-92. 